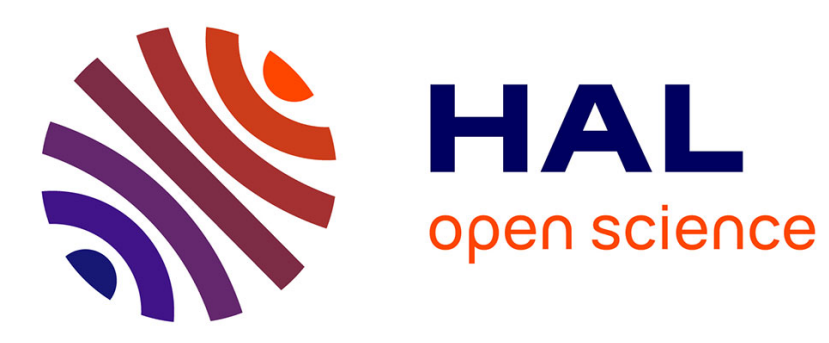

\title{
Crystallization kinetics in glassy GexSe100 - x
}

S. Goel, S.K. Tripathi, A. Kumar

\section{To cite this version:}

S. Goel, S.K. Tripathi, A. Kumar. Crystallization kinetics in glassy GexSe100 - x. Revue de Physique Appliquée, 1990, 25 (4), pp.333-338. 10.1051/rphysap:01990002504033300 . jpa-00246191

\section{HAL Id: jpa-00246191 https://hal.science/jpa-00246191}

Submitted on 1 Jan 1990

HAL is a multi-disciplinary open access archive for the deposit and dissemination of scientific research documents, whether they are published or not. The documents may come from teaching and research institutions in France or abroad, or from public or private research centers.
L'archive ouverte pluridisciplinaire HAL, est destinée au dépôt et à la diffusion de documents scientifiques de niveau recherche, publiés ou non, émanant des établissements d'enseignement et de recherche français ou étrangers, des laboratoires publics ou privés. 


\title{
Crystallization kinetics in glassy $\mathrm{Ge}_{x} \mathrm{Se}_{100-x}$
}

\author{
S. Goel, S. K. Tripathi and A. Kumar \\ Department of Physics, Harcourt Butler Technological Institute, Kanpur - 208002 , India
}

(Reçu le 25 novembre 1988, révisé le 17 août 1989, accepté le 18 décembre 1989)

\begin{abstract}
Résumé. - La cinétique de cristallisation du système $\mathrm{Ge}_{x} \mathrm{Se}_{100-x}$ vitreux, est étudiée en utilisant la technique isothermique, c'est-à-dire, en étudiant la transformation de la phase amorphe à la phase cristalline par recuit isotherme à différentes températures entre la transition vitreuse et la température de fusion. On choisit la conductivité en courant continu comme paramètre caractéristique pour mesurer le taux de cristallisation durant le processus de cristallisation. Pour calculer l'énergie d'activation de cristallisation et le paramètre d'ordre, les résultats expérimentaux sont ajustés selon l'équation d'Avrami pour une transformation isotherme. Les paramètres de la cinétique calculés, indiquent que différents mécanismes de cristallisation sont prépondérants dans les différents domaines de transformation. Les valeurs relatives des paramètres de la cinétique sont discutées selon la structure du système Ge-Se.
\end{abstract}

\begin{abstract}
Crystallization kinetics of glassy $\mathrm{Ge}_{x} \mathrm{Se}_{100-x}$ system is studied using isothermal technique, i.e., by studying amorphous to crystalline transformation during isothermal annealing at various temperatures between glass transition and melting temperature. DC conductivity is taken a characteristic quantity to measure the extent of crystallization during crystallization process. To calculate the activation energy of crystallization and the order parameter, the data is fitted to the Avrami's equation of isothermal transformation. The calculated kinetic parameters indicate that different crystallization mechanisms are dominant in various ranges of transformations. The composition dependence of kinetic parameters is also discussed in terms of the structure of Ge-Se system.
\end{abstract}

\section{Introduction.}

Chalcogenide glasses have recently drawn great attention of scientists due to their applications in various solid state devices. The materials are well known for their use in threshold and memory switching. A particular glassy alloy will behave as a threshold or memory switch depends upon the rate of crystallization [1]. Those materials which have higher crystallizing tendency show memory switching. The study of crystallization kinetics is, therefore, an important issue for chalcogenide glasses. Apart from the above technological importance, the knowledge of the crystallization processes is important for the better understanding of the short range order in these materials. The present paper reports the crystallization studies in one of the important glassy chalcogenide system $\mathrm{Ge}_{x} \mathrm{Se}_{100-x}$ where $5 \leqslant x \leqslant 22$.

The extent of crystallinity $(\alpha)$ of a certain material which crystallizes isothermally with time $(t)$ is represented by the Avrami's equation [2] as follows :

$$
\alpha(t)=1-\exp \left(-K t^{n}\right)
$$

where $K$ is the rate constant and $n$ is an order parameter which depends upon the mechanism of the crystal growth.

The temperature dependence of $K$ can be represented by an Arrhenius equation as :

$$
K=K_{0} \exp \left(-\frac{\Delta E}{k T}\right)
$$

where $K_{0}$ is a constant and $\Delta E$ represents the activation energy of crystallization.

Any physical quantity which changes drastically upon crystallization can be used to understand the extent of crystallinity $(\alpha)$ and hence kinetic parameters $(\Delta E$ and $n)$ can be calculated using the above equations. DC conductivity has been used to study crystallization kinetics in chalcogenide glasses by Kotkata et al. [3-6].

Odelevsky [7] has suggested a power formula to calculate the conductivity of a mixture $\left(\sigma_{\mathrm{m}}\right)$ during amorphous to crystalline transformation. According to him

$$
\sigma_{\mathrm{m}}^{\ell}=\alpha \sigma_{\mathrm{c}}^{\ell}+(1-\alpha) \sigma_{\mathrm{a}}^{\ell}
$$


where $\sigma_{c}$ and $\sigma_{a}$ are the conductivities of the crystalline and amorphous phases having volume fractions $\alpha$ and $1-\alpha$ respectively.

For the power $(\ell)=1$, the measured conductivity $\left(\sigma_{\mathrm{m}}\right)$ at a particular time during crystallization can be written as

$$
\sigma_{\mathrm{m}}=\alpha \sigma_{\mathrm{c}}+(1-\alpha) \sigma_{\mathrm{a}} .
$$

On the other hand, when $\log \sigma$ is considered to represent the sensitive parameter characterizing the conductivity-content dependence, an empirical power formula may be written as

$$
\log \sigma_{\mathrm{m}}=\alpha \log \sigma_{\mathrm{c}}+(1-\alpha) \log \sigma_{\mathrm{a}} .
$$

Kotkata et al. [6] have used equations (4) and (5) to calculate the extent of crystallization in some $\mathrm{Se}$ based chalcogenide glasses and found that equation (5) is more satisfactory as $\left(\sigma_{c}-\sigma_{a}\right)$ is quite large in these glasses.

In the present study we have used equation (5) to study the crystallization kinetics in glassy $\mathrm{Ge}_{x} \mathrm{Se}_{100-x}$ for $x=5,10,15$ and 22 . The results indicate that the different crystallization mechanisms are dominant in various ranges of transformations. The kinetic parameters are also found to be composition dependent which is explained in terms of the structure of $\mathrm{Ge}-\mathrm{Se}$ system.

Section 2 describes the experimental details. The results have been presented in section 3 and discussed in section 4 . The last section deals with the conclusions of the present work.

\section{Experimental details.}

Glassy alloys of $\mathrm{Ge}_{x} \mathrm{Se}_{100-x}$ with $x=5,10,15$ and 22 were prepared by quenching technique. $99.999 \%$ pure materials were sealed in quartz ampoules with a vacuum $\sim 10^{-5}$ torr. The ampoules were kept inside a furnace where the temperature was raised slowly $\left(3-4{ }^{\circ} \mathrm{C}\right.$ per minute) to $950{ }^{\circ} \mathrm{C}$. The ampoules were rocked frequently for 10 hours at the maximum temperature and the quenching was done in air by an air blower.

The glassy alloys thus prepared were grounded to a very fine powder and the pellets (dia $\sim 6 \mathrm{~mm}$ and thickness $\sim 0.5-1 \mathrm{~mm}$ ) were obtained after compressing the powder in a die at a pressure of 3-4 tons.

The amorphous to crystalline transformation (a-c) was studied by measuring the dc conductivity $(\sigma)$ as a function of time $(1 / 2 \mathrm{~min}$ intervals) at various temperatures. The temperature was between $T_{\mathrm{g}}$ (the glass transition temperature) and $T_{\mathrm{m}}$ (the melting temperature) and was kept constant during the amorphous to crystalline transformation period.

The conductivity measurements were taken in a vacuum $\sim 10^{-2}$ torr by mounting the samples in a specially designed metallic sample holder. The current was measured using Keithley Electrometer (model 614). The temperature was measured using calibrated cromel-alumel thermocouple.

Uncoated pellets were preferred to avoid any electrode migration at high temperatures (upto $270^{\circ} \mathrm{C}$ ). Though the uncoated pellets opens a possibility of poor electrical contact, the conclusions of the present work will not be affected much as all the measurements are done under the same conditions. Different pellets were taken for each temperature of annealing. The annealing temperature was obtained at a fast heating rate and then maintained constant for quite sometime till a saturation in current value is reached with time.

\section{Results.}

At any isothermal annealing between $T_{\mathrm{g}}$ and $T_{\mathrm{m}}$, the electrical conductivity $\sigma$ varies with the annealing time $t$. Figure 1 shows the variation in $\log \sigma$ with the annealing time $t$ in glassy $\mathrm{Ge}_{5} \mathrm{Se}_{95}$ and $\mathrm{Ge}_{22} \mathrm{Se}_{78}$ systems annealed at different temperatures. The results for other compositions were also found to be of the same nature. During the transformation process, there appears to be three regimes of $\sigma$ versus percentage transformation $\alpha$ (see Fig. 1). The part $A B$ of the plots are linear and represents a gradual increase in $\sigma$ as a result of the normal heating of the amorphous samples. The amorphous to crystalline transformation has a two step character, i.e., it passes through two distinguish-
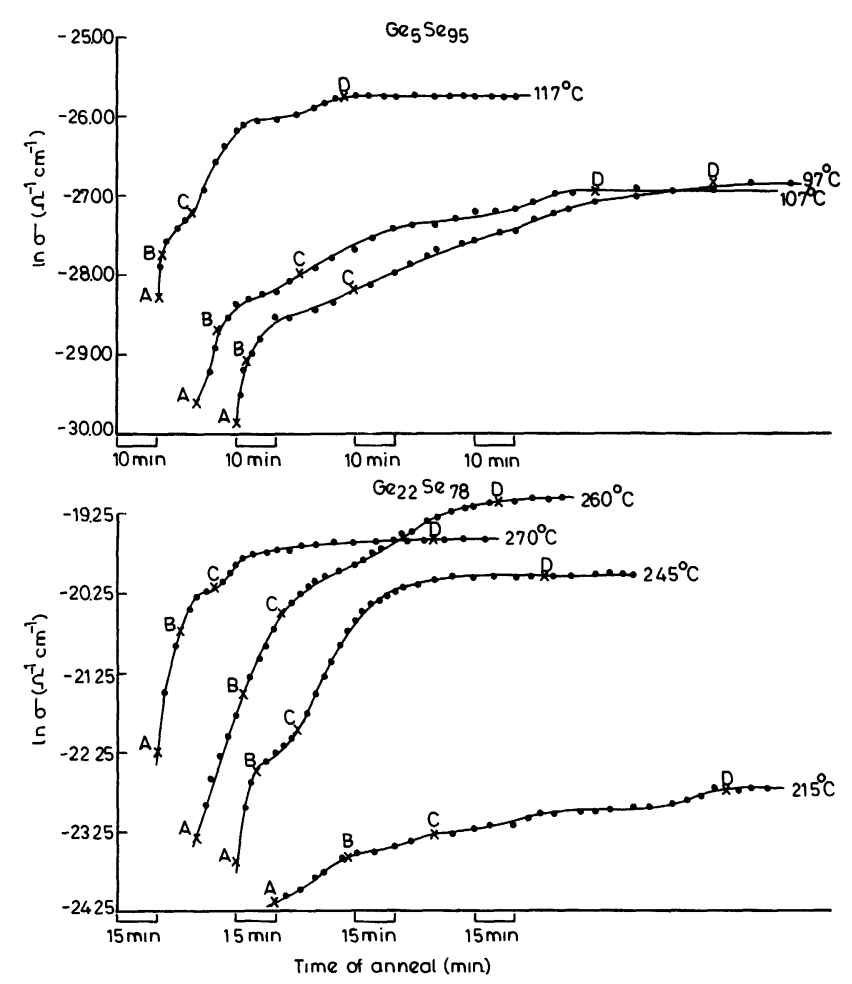

Fig. 1. - Annealing time dependence of dc conductivity for $\mathrm{Ge}_{5} \mathrm{Se}_{95}$ and $\mathrm{Ge}_{22} \mathrm{Se}_{78}$ samples during different isothermal amorphous-crystal phase transformations. 
able time dependent stages, $\mathrm{BC}$ and $\mathrm{CD}$. $\mathrm{CD}$ attains a limiting value (point $\mathrm{D}$ ) after a certain time.

The increase in annealing temperature enhances the normal heating process, leads to a decrease in its duration and the onset of the next stage (crystallization) after a shorter time. It was observed that increasing the temperature of annealing leads to a decrease in the total time $(\tau)$ necessary for completing the transformation process.

The proper heat treatment of an amorphous alloy leads, generally, to the production of materials having different ratios of crystalline and amorphous fractions. So, at intermediate point during the a-c transition, the measured values of $\sigma(t)$ represents the contribution of two conductivities of two mixed phases: the amorphous phase of conductivity $\sigma_{\mathrm{a}}$ (Fig. 1 points $\mathrm{C}$ ) and the crystalline phase of conductivity $\sigma_{c}$ (Fig. 1 points D). However, the less pronounced increase of $\sigma$ during the first stage, AB is mainly accompanied by the formation of dispersed centers (or nuclei) and the growth of these electrically isolated nuclei at the expense of the parent amorphous phase. Also, the second stage $\mathrm{BC}$, which covers a relatively large increase in $\sigma$, indicates the subsequent crystal growth of the new phase till complete transformation of sample volume is attained. This large increase in $\sigma$ is associated with the possibility of binding the nuclei during the growth of the new phase and finally producing non-isolated crystallites extended through the sample.

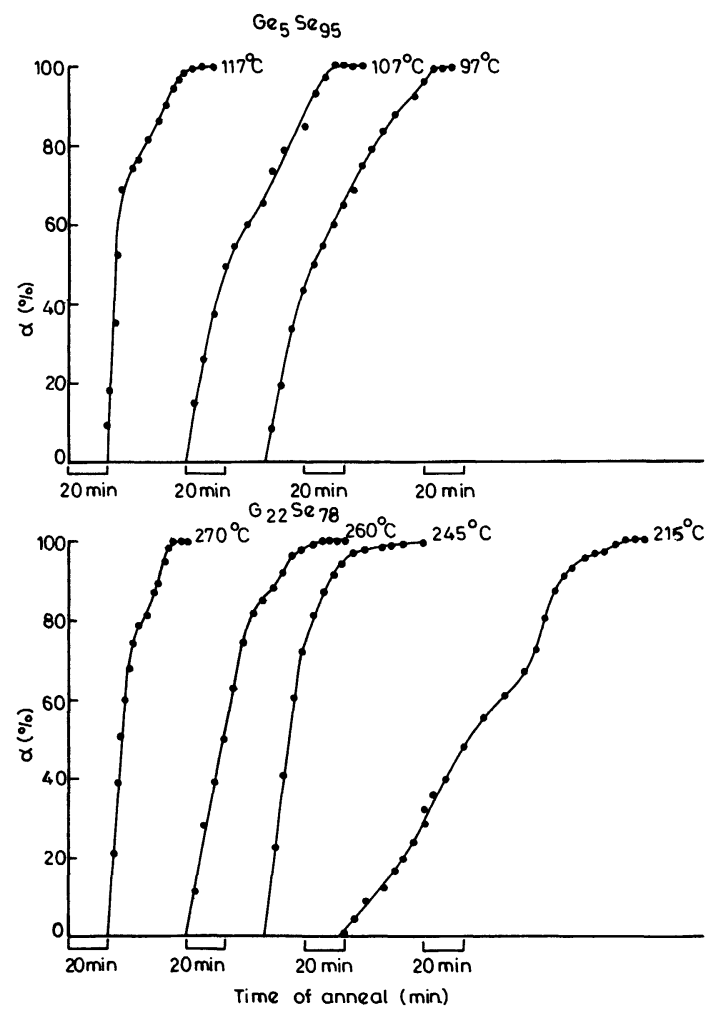

Fig. 2. - Crystallinity percentage vs. annealing time for $\mathrm{Ge}_{5} \mathrm{Se}_{95}$ and $\mathrm{Ge}_{22} \mathrm{Se}_{78}$ crystallized for different isotherms.
The transformed fraction $\alpha$ as a function of $t$ is computed for the $\mathrm{Ge}_{x} \mathrm{Se}_{100-x}$ on the basis of equation (5). Figure 2 shows the time dependence of $\alpha$ for $\mathrm{Ge}_{5} \mathrm{Se}_{95}$ and $\mathrm{Ge}_{22} \mathrm{Se}_{78}$ during growth for different annealing temperatures. The results for the other compositions were also found to be of the same nature. The value of $\alpha$ at any time $t$ is related to the transformation kinetic parameters by the Avrami equation (Eq. (1)). From the slope of $\ln [-\ln (1-\alpha)] v s . \ln t$ curves one can calculate the value of $n$.

Figure 3 shows the so-called Avrami plot in case of $\mathrm{Ge}_{x} \mathrm{Se}_{100-x}$ for different isothermal anneals. The resulting curves have shapes similar to that usually obtained for the crystallization kinetics of other materials [4-6]. In this case the plot has more than one slope during the amorphous to crystalline transformation which therefore can have different values of $n$.

Table I gives the variation of $n$ at different temperatures as a function of sample composition. A change in the exponent $n$ indicates a change in growth mechanism during crystallization. According to Evans [8], there is a linear change in the number of nuclei with time, with an increase in the rate of increase of nuclei. The exponent $n$ can acquire increasing values, i.e., $\boldsymbol{n}$ can have continuous values upto 4 instead of 3 for sporadic three dimensional growth. For a predetermined process, $n$ can take only three integer values, 1,2 or 3 , depending on whether it represents one, two or three dimensional growth respectively.

In the present case also the value of $n$ and $\Delta E$ is different in different temperature ranges. This indicates that different crystallization mechanisms

Table I. - Values of $n$ at various annealing temperatures.

\begin{tabular}{lcccc}
\hline Composition & & & & \\
& $97{ }^{\circ} \mathrm{C}$ & $107{ }^{\circ} \mathrm{C}$ & $117^{\circ} \mathrm{C}$ & \\
$\mathrm{Ge}_{5} \mathrm{Se}_{95}$ & $n_{1} 1.11$ & 1.00 & 0.91 & \\
& $n_{2} 0.48$ & 0.15 & 0.29 & \\
& $n_{3} 1.42$ & 1.28 & 1.23 & \\
& $137{ }^{\circ} \mathrm{C}$ & $147^{\circ} \mathrm{C}$ & $157^{\circ} \mathrm{C}$ & $167^{\circ} \mathrm{C}$ \\
$\mathrm{Ge}_{10} \mathrm{Se}_{90}$ & $n_{1} 0.71$ & 0.77 & 0.86 & 0.80 \\
& $n_{2} 1.54$ & 2.06 & 2.28 & 2.30 \\
& $195{ }^{\circ} \mathrm{C}$ & $205^{\circ} \mathrm{C}$ & $22{ }^{\circ} \mathrm{C}$ & $225^{\circ} \mathrm{C}$ \\
$\mathrm{Ge}_{15} \mathrm{Se}_{85}$ & $n_{1} 0.98$ & 1.03 & 0.77 & 0.80 \\
& $n_{2} 0.49$ & 0.50 & 0.51 & 0.66 \\
& $n_{3} 1.33$ & 1.23 & 1.21 & 1.20 \\
& $215{ }^{\circ} \mathrm{C}$ & $245^{\circ} \mathrm{C}$ & $260{ }^{\circ} \mathrm{C}$ & $270{ }^{\circ} \mathrm{C}$ \\
$\mathrm{Ge}_{22} \mathrm{Se}_{78}$ & $n_{1} 1.33$ & 1.20 & 1.49 & 1.32 \\
& $n_{2} 0.35$ & 0.54 & 0.88 & 0.38 \\
& $n_{3} 1.47$ & 2.07 & 1.61 & 2.00 \\
\hline
\end{tabular}



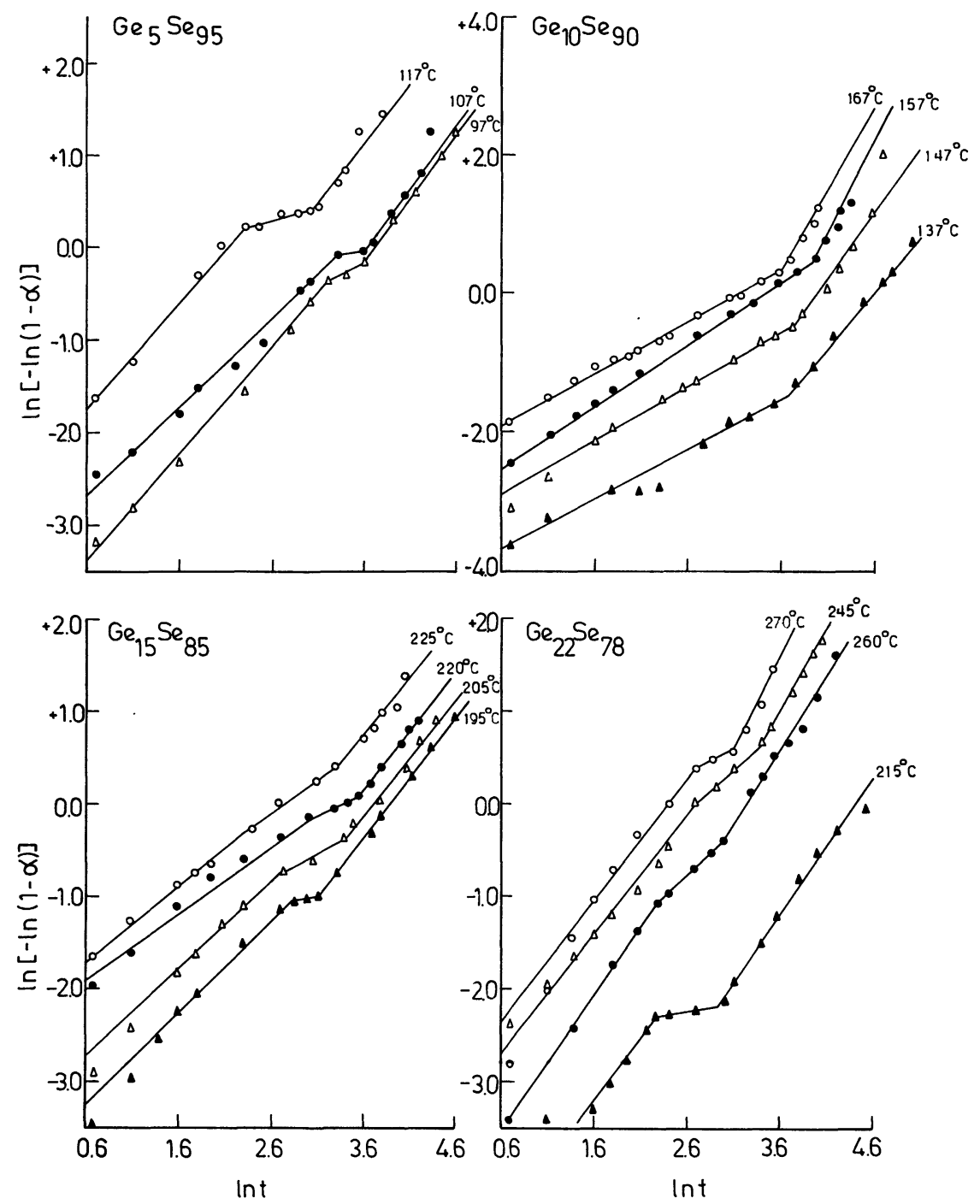

Fig. 3. - Avrami plots of the crystallization of $\mathrm{Ge}_{x} \mathrm{Se}_{100-x}$ for different isotherms.

are dominant in various ranges of transformation, where germanium affects the crystallization of selenium in different ways. The exact mechanisms, however, can not be predicted from the present measurements only.

The values of temperature dependent crystallization rate constant were calculated for each value of $\alpha$ during the equation (1)

$$
K=\frac{\ln (1-\alpha)^{-1}}{t^{n}}
$$

The dependence of $\ln K$ on $\frac{1}{T}$ is given in figure 4 . The plot yields a straight line relationship and the values of the crystallization activation energy $\Delta E$ for the different stages of transformation are given in table II.

\section{Discussions.}

On the basis of various physical properties, covalently bonded amorphous semiconductors can be devided into two main categories (1) Ge type which includes tetrahedrally co-ordinated substances, e.g., $\mathrm{Ge}, \mathrm{Si}$ and III-V compounds (ii) Se type which includes the chalcogen elements ( $\mathrm{S}, \mathrm{Se}$ and $\mathrm{Te}$ ) and their multicomponent alloys. $\mathrm{Ge}_{x} \mathrm{Se}_{100-x}$ system is expected to fall into either of the category depending upon the value of $x$. Street and Biegelsen [9] studied the optical gap and photoluminescence in $\mathrm{Ge}_{x} \mathrm{Se}_{100-x}$ system and found the properties to be composition dependent. Various other physical properties such as drift mobility [10], photocontraction [11], dc conduction [12, 13], elastic properties [14], X-ray studies [15] and photoconducting properties [16] have also been studied in various laboratories. These properties have also been found to be highly composition dependent.

In the present case also, the values of $\Delta E$ are highly composition dependent (see Tab. II). The activation energy of crystallization (I process) decreases as the Ge concentration increases in $\mathrm{Ge}_{x} \mathrm{Se}_{100-x}$ system. The decreasing value of $\Delta E$ 
Table II. - Activation energy $\Delta E$ of the crystallization processes, as calculated from slopes of the relation $\ln K$ versus $T^{-1}$.

\begin{tabular}{lccc}
\hline \multirow{3}{*}{ Composition } & \multicolumn{3}{c}{$\Delta E(\mathrm{eV})$} \\
\cline { 2 - 4 } & $\Delta E_{1}$ & $\Delta E_{2}$ & $\Delta E_{3}$ \\
\hline $\mathrm{Ge}_{5} \mathrm{Se}_{95}$ & 1.52 & 1.38 & 1.42 \\
$\mathrm{Ge}_{10} \mathrm{Se}_{90}$ & 1.07 & - & 0.43 \\
$\mathrm{Ge}_{15} \mathrm{Se}_{85}$ & 1.02 & 0.75 & 0.90 \\
$\mathrm{Ge}_{22} \mathrm{Se}_{78}$ & 0.93 & 0.84 & 0.41 \\
\hline
\end{tabular}

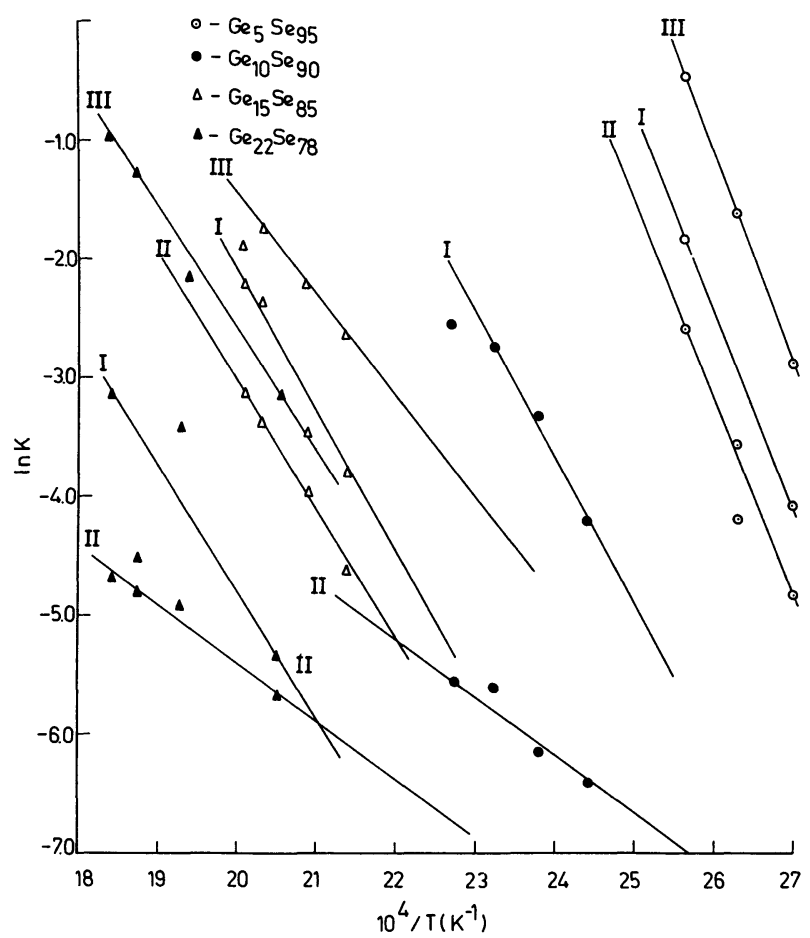

Fig. 4. - Arrhenius plots for different stages of crystallization of $\mathrm{Ge}_{x} \mathrm{Se}_{100-x}$.

suggests that the crystallization becomes slower and slower as Ge concentration increases which may be understood as follows :
According to Phillips [17], the molecular structure of melt quenched $\mathrm{Ge}_{x} \mathrm{Se}_{100-x}$ is much more ordered than would be expected from a continuous random network model. According to him, Ge-Se alloys may be described by small chemically ordered clusters embedded in a continuous network. Some of these clusters are $(\mathrm{Se})_{n}$ chains, $\mathrm{Ge}\left(\mathrm{Se}_{1 / 2}\right)_{4}$ corner sharing tetrahedra and $\left(\mathrm{Ge}_{2} \mathrm{Se}_{1 / 2}\right)_{6}$ ethane-like structural units.

At very low concentration of $\mathrm{Ge},(\mathrm{Se})_{n}$ chains predominates and the crystallization process may be fast as the structural modifications are easier in twofold co-ordination. However, as $\mathrm{Ge}$ concentration increases, $\mathrm{Ge}\left(\mathrm{Se}_{1 / 2}\right)_{4}$ clusters increases on the expense of $(\mathrm{Se})_{n}$ chains. As tetrahedral structure is more rigid, the crystallization processes may become slow at higher concentration of Ge. At much higher concentrations of $\mathrm{Ge},\left(\mathrm{Ge}_{2} \mathrm{Se}_{1 / 2}\right)_{6}$ ethane-like structure dominates. But, the present measurements coult not be extended for such a high percentage of Ge $(x>33)$ as we could not pelletize the powder at higher $\mathrm{Ge}$ concentration.

\section{Conclusions.}

The amorphous to crystalline transformation processes have been studied in the four different compositions of a binary system $\mathrm{Ge}_{x} \mathrm{Se}_{100-x}(x=5$ to 22). The electrical conductivity is taken as a characteristic quantity to follow the growth of crystalline phases in the amorphous matrix. By fitting the experimental data to the Avrami's equation of transformation kinetics, the kinetic parameters have been calculated at various temperatures. The results indicate that different crystallization mechanisms dominate in various ranges of transformations. Moreover, the percentage of Ge plays an important role in the crystallization processes. This is in agreement with the structure of $\mathrm{Ge}_{x} \mathrm{Se}_{100-x}$ system.

\section{Acknowledgments.}

We are grateful to Prof A. N. Nigam for his help in the experimental set up. This work was supported by a project sanctioned by University Grants Commission, New Delhi (India).

\section{References}

[1] Kumar A., Ph. D. thesis, Panjab University (1979).

[2] Avrami M., J. Chem. Phys. 8 (1940) 212.

[3] Kotkata M. F., El-Dib A. F. and Gani F. A., Mater. Sci. Eng. 72 (1985) 163.

[4] Kotkata M. F. and El-Mously M. K., Acta Phys. Hungarica 54 (1983) 303.

[5] Kotkata M. F., Kamal G. M. and El-Mously M. K., Indian J. Technol. 20 (1982) 390.

[6] Kotkata M. F., El-Mously M. K. and Ayad F. M., Acta Phys. Hungarica 46 (1979) 19.
[7] Odelevsky V. I., J. Techn. Phys. USSR 21 (1951) 673.

[8] Evans U. R., Trans. Faraday Soc. 41 (1945) 365.

[9] STREet R. A. and Biegelsen D. K., J. Non-cryst. Solids 32 (1979) 339.

[10] Kim G. I. and Shirafugi J., Jpn J. Appl. Phys. 17 (1984) 71.

[11] Singh B., Rajagopalan S., Bhat P. K., Pandaya D. K. and ChOprA K. L., Solid State Commun. 29 (1979) 167. 
[12] Frank S. and Owen A. E., Phys. Status Solidi (a) 16 (1973) 623.

[13] Mehra R. M., Kumar H., Agarwal S. C., Sikka P. and MATHUR P. C., J. Non-cryst. Solids 77 \& 78 (1985) 1241.

[14] Mahadevan S., Giridhar A., Singh A. K., J. Non-cryst. Solids 57 (1983) 423.
[15] Agnihotri A. K., Kumar A. and Nigam A. N., Philos. Mag. Lett. 58 (1988) 63.

[16] Kumar A., Goel S. and Tripathi S. K., Phys. Rev. B 38 (1988) 13432.

[17] Phillips J. C., J. Non-cryst. Solids 43 (1981) 37. 